\title{
Analysis of holographic data storage using a PA-LCoS device
}

\author{
Francisco J. Martínez ${ }^{1,2}$, Andrés Márquez ${ }^{1,2,{ }^{*}}$, Sergi Gallego ${ }^{1,2}$, Sandra Fenoll ${ }^{1,2}$, Manuel Ortuño ${ }^{1,2}$, \\ Jorge Francés ${ }^{1,2}$, Sergio Bleda ${ }^{1,2}$, Inmaculada Pascual ${ }^{2,3}$ \\ ${ }^{1}$ Dept. de Física, Ing. de Sistemas y T. Señal, Univ. de Alicante, Ap. 99, E-03080, Alicante, Spain \\ ${ }^{2}$ I.U. Física Aplicada a las Ciencias y las Tecnologías U. de Alicante, Ap. 99, E-03080, Alicante, Spain \\ ${ }^{3}$ Dept. de Óptica, Farmacología y Anatomía, Univ. de Alicante, Ap. 99, E-03080, Alicante, Spain
}

\begin{abstract}
Holographic data storage systems (HDSS) have been a promising and very appealing technology since the first laser developments in the sixties. Impact of ongoing advances in the various components needs to be explored in its specific application to HDSS. In this sense, continuous progress is being produced in spatial light modulator (SLM) technology where parallel-addressed liquid crystal on silicon (PA-LCoS) microdisplays have replaced previous liquid-crystal displays (LCD) in most of optics and photonics applications. PA-LCoS microdisplays are well adapted to display phaseonly elements without coupled amplitude. In this paper, we analyse how PA-LCoS devices can also be used to display the widely applied binary intensity modulated (BIM) data pages. We also investigate hybrid-ternary modulated (HTM) data pages, which are very much demanding on the phase and amplitude modulation properties of an SLM. HTM data pages combine the ease of detection of BIM data pages, together with a large reduction of the DC term of the Fourier Transform of the data page. This reduction is necessary to avoid saturation of the recording material dynamic range. Simulated results show the magnitude of the expected DC term in the Fourier plane. We have verified the good performance of PA-LCoS to display BIM data pages. We have also obtained that pure HTM data pages cannot be produced with PA-LCoS devices, however, a rather close performance is obtained when implementing the pseudo-HTM data pages. In this work a more complete study of pseudo-HTM modulation is offered.
\end{abstract}

Keywords: Holographic and volume memories, liquid crystal on silicon displays, parallel aligned, spatial light modulation.

\section{INTRODUCTION}

Holographic data storage systems (HDSS) need an entry data point. They use a spatial light modulator (SLM) that allows to change the data dynamically. Parallel aligned liquid crystal on silicon devices (PA-LCoS) have been widely used as SLM. They offer an easy operation as phase-only device, and they can be easily used as amplitude only devices. These features bring us the opportunity to select the modulation regime required by our application. PA-LCoS have been found useful in many applications as in diffractive optics ${ }^{[1]}$, optical storage ${ }^{[2]}$, optical metrology ${ }^{[3]}$, reconfigurable interconnects $^{[4][5]}$, or quantum optical computing ${ }^{[6]}$, due to their very high spatial resolution and very high light efficiency ${ }^{[7][8]}$.

PA-LCoS displays can be assimilated to linear variable retarders ${ }^{[7][8]}$, but they exhibit some flicker or fluctuations ${ }^{[9]-[14]}$, specially digitally addressed backplane devices ${ }^{[15][16]}$ which are widely used. Then, for a full characterization of the PALCoS device, the retardance and the fluctuation amplitude will be needed. To this goal some techniques, based on simple polarimeter setups, were developed by our group and Ramirez et al. ${ }^{[17]-[19]}$. A more detailed characterization was obtained by applying time-average Stokes polarimetry ${ }^{[20]}$. Using a Mueller based model we can predict the response of the device for every gray level and any kind of state of polarization (SOP) at the system entry.

In the present work, this method is used to predict the performance of a specific modulation scheme for data pages. We analyse how to use PA-LCoS devices to display binary intensity modulated (BIM) data pages. We also investigate hybrid-ternary modulated (HTM) data pages, which are very much demanding on the phase an amplitude modulation properties of an SLM. We have verified the good performance of PA-LCoS to display BIM data pages, and we have also observed that pure HTM data pages cannot be implemented with PA-LCoS devices, however, a rather close performance

Optical Modelling and Design IV, edited by Frank Wyrowski, John T. Sheridan, Youri Meuret, Proc. of SPIE Vol. 9889, $988922 \cdot$ (C) 2016 SPIE · CCC code: 0277-786X/16/\$18 · doi: 10.1117/12.2227948 
is obtained when implementing the proposed pseudo-HTM scheme that we recently proposed and which we have here further analysed ${ }^{[21]}$.

\section{MODULATION SCHEMES}

In order to use a PA-LCoS device for HDSS a modulation scheme has to be selected. PA-LCoS devices are well adapted to display phase-only elements since they enable phase-only modulation of the object beam without coupled amplitude. This can be achieved when the device is illuminated by linearly polarized light parallel to the director axis. In this section, we investigate that PA-LCoS devices can also be used to display the widely applied BIM data pages and HTM data pages. HTM modulation scheme is very much demanding on the phase and amplitude modulation properties of the device used as SLM, and PA-LCoS have not yet been studied when HTM scheme is used.

In this section we use a novel characterization technique to obtain the linear retardance and flicker amplitude introduced by the device as a function of gray level ${ }^{[20][22]}$. Then, we configure and calibrate our device and we use these values to calculate the complex amplitude modulation of the PA-LCoS when it is inserted between external polarization elements (polarizers and/or waveplates).

\subsection{Jones Matrix Formalism}

In our previous works, we were interested in the state of polarization (SOP) and the degree of polarization (DoP) at the device exit, so we used Mueller-Stokes formalism. In order to analyze the phase-shift and amplitude modulation capabilities of the PA-LCoS devices, Jones matrix formalism is a better choice. Next, we provide the basic expressions necessary for the complex amplitude calculations. Equation (1) represents the matrix associated with a linear polarizer with its transmission axis along the $\mathrm{X}$-axis, which we consider along the vertical of the lab,

$$
P x=\left(\begin{array}{ll}
1 & 0 \\
0 & 0
\end{array}\right)
$$

The matrix for a linear retarder of linear retardance $\varphi$ with its slow axis along the $\mathrm{X}$-axis in the reference system is given by:

$$
W(\varphi)=\left(\begin{array}{cc}
\exp (-j \varphi / 2) & 0 \\
0 & \exp (+j \varphi / 2)
\end{array}\right)
$$

If the polarization elements are rotated with respect to the $\mathrm{X}$-axis by an angle $\theta$, the rotation matrix is necessary:

$$
R(\theta)=\left(\begin{array}{cc}
\cos \theta & \sin \theta \\
-\sin \theta & \cos \theta
\end{array}\right)
$$

In order to produce the two mentioned modulation schemes, BIM and HTM, with the PA-LCoS, we inserted the device between two rotated polarizers. With this setup, we can calculate the complex amplitude for the electric field at the exit, $\vec{E}_{\text {OUT }}$, using equation (4):

$$
\vec{E}_{\text {OUT }}=P_{X} \cdot R\left(\theta_{2}\right) \cdot W_{P A}(\bar{\Gamma}) \cdot\left(\begin{array}{c}
\cos \theta_{1} \\
\sin \theta_{1}
\end{array}\right)
$$

where $\theta_{1}$ and $\theta_{2}$ are the angles of the linear polarizers at the system entry and exit respectively, with respect to the Xaxis. The expression $W_{P A}(\bar{\Gamma})$ is the matrix for the PA-LCoS device, as the device can be considered a variable linear retarder. The expression of a linear retarder is defined by equation (2), where $\bar{\Gamma}$ can be changed by the voltage applied and it is measured with the average Stokes polarimetric technique developed by our group ${ }^{[20]}$. 


\subsection{Configuring PA-LCoS}

The specific PA-LCoS device considered in this work is a commercially available PA-LCoS microdisplay, model PLUTO distributed by the company HOLOEYE. It is filled with a nematic liquid crystal, with 1920x1080 pixels and 0.7 " diagonal. The pixel pitch is of $8.0 \mu \mathrm{m}$ and the display has a fill factor of $87 \%$. The signal is addressed via a standard DVI (Digital Visual Interface) signal. By means of the RS-232 interface and its corresponding provided software, we have access to the basic electrical parameters of the device ${ }^{[10][11]}$, such as the digital addressing sequence format, the gamma curve, and the voltage dynamic range of the pulse width modulated (PWM) signal (through two digital potentiometers), i.e. the so-called voltages $\mathrm{V}_{\text {bright }}$ and $\mathrm{V}_{\text {dark }}$. These $\mathrm{V}_{\text {bright }}$ and $\mathrm{V}_{\text {dark }}$ voltages express the maximum and minimum amplitudes of the pulsed voltage applied across the LC layer. In particular the software for the SLM is provided with a series of configuration files corresponding to two different digital addressing sequences: the so-called 18-6 and 5-5 digital sequences. The first number indicates the quantity of "equally weighted" bit-planes, and the second number the quantity of "binary" bit-planes ${ }^{[10]}$. This means that the sequence $18-6$ is longer than the one corresponding for the sequence 5-5. In principle the shorter the sequence the smaller the flicker ${ }^{[10]}$ thus in the following we restrict our attention to sequence 5-5 since it produces less flicker.

In order to obtain the relation of the retardance as a function of gray level we have to select a specific setup in the lab. We used the next experimental setup:

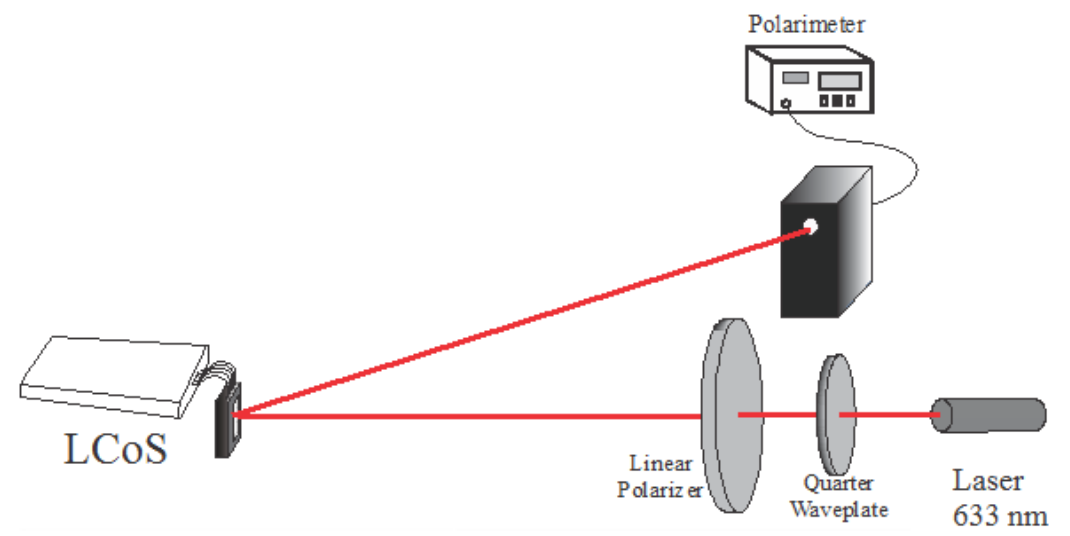

Fig. 1. Experimental setup used to measure linear retardance and flicker with the averaging Stokes polarimetric technique: Incidence at $11.5^{\circ}$.

Retardance measurements have been taken at an angle of incidence of $11.5^{\circ}$, and for the wavelength $532 \mathrm{~nm}$. These are the working conditions of the PA-LCoS device in the HDSS setup that we use. The PA-LCoS device is addressed with two different electrical configurations adapted in each case for the retardance dynamic range necessary for BIM and for HTM and minimizing the possibility of flicker. We know how to adapt the electrical configuration as a function of the application $^{[16]}$. In this sense we consider different pair of values for the configuration voltages $\mathrm{V}_{\text {bright }}$ and $\mathrm{V}_{\text {dark: for }}$ BIM and for HTM they are respectively $\left(\mathrm{V}_{\text {bright }}=2.02 \mathrm{~V}, \mathrm{~V}_{\text {dark }}=1.11 \mathrm{~V}\right)$ and $\left(\mathrm{V}_{\text {bright }}=3.82 \mathrm{~V}, \mathrm{~V}_{\text {dark }}=0.03 \mathrm{~V}\right)$. These values enable to produce a $\pi$ radians retardance range for BIM, which is enough to generate an amplitude transmission configuration with minimum and maximum intensity transmission values for the specified wavelength. In the case of HTM, we consider voltages producing a larger retardance range, close to $2 \pi$ radians, since we not only need a low and high transmission intensity values, but we also need two equally high transmission intensity points with a relative phaseshift difference as close to $\pi$ radians as possible. In the case of twisted-nematic LCDs, we showed that these conditions were better met if the modulation dynamic range available is increased ${ }^{[25]-[28]}$. In this section we analyse if this is still the case with PA-LCoS devices.

Taking into account the setup described we obtain the retardance and the flicker amplitude as a function of gray level represented in figure 2. This figure shows the configuration for BIM in continuous line and for HTM in dashed line. We note that for BIM the average retardance range is slightly above $180^{\circ}$ and for HTM is above $360^{\circ}$. Due to the different configurations we observe a different behavior of the fluctuation amplitude: is about $10^{\circ}$ for the BIM configuration and reaches $40^{\circ}$ in the HTM configuration, which is due to the changes performed in $\mathrm{V}_{\text {bright }}$ and $\mathrm{V}_{\text {dark }}^{[16]}$. 


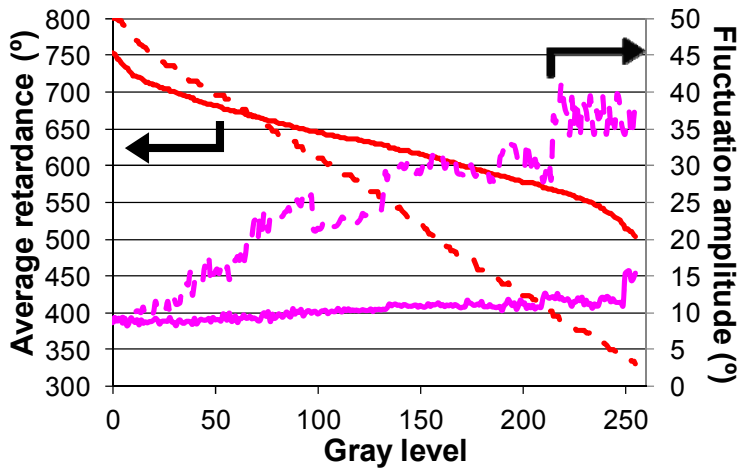

Fig. 2. Calculated values for the average retardance and the fluctuation amplitude for $\lambda=532 \mathrm{~nm}$, at an angle of incidence of $11.5^{\circ}$, for sequence $5-5$ with configuration voltages $($ Vbright $=2.02 \mathrm{~V}$, Vdark $=1.11 \mathrm{~V})$ for BIM (continuous) and $($ Vbright $=3.82 \mathrm{~V}$, Vdark $=0.03 \mathrm{~V}$ ) for HTM (dashed).

\subsection{Binary Intensity Modulation}

The objective in a BIM scheme is to generate the maximum intensity contrast between the ON and OFF values. If contrast is defined as $I_{\text {contrast }}=I_{O N} / I_{O F F}$, an acceptable raw BER (Bit Error Rate) can be obtained ${ }^{[30]}$ with a contrast of 1:20. We can obtain the maximum contrast just using linear polarizers. The transmission axes of the polarizers have to be oriented at $45^{\circ}$ angle with respect to the director axis of the PA-LCoS and they must be parallel or crossed with respect to each other. Taking into account the values showed in figure 2 and equation (4) we obtain the intensity transmission and the phase-shift curves as a function of gray level that are represented in fig. 3, which also shows the phasor representation in the complex plane.

(a)

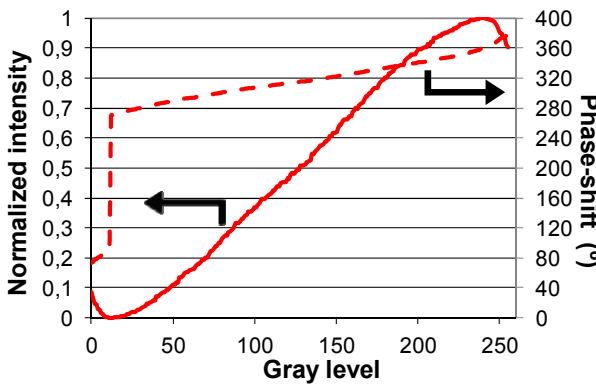

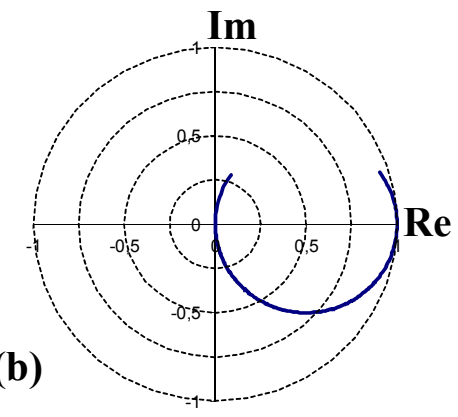

Fig. 3. Simulation for BIM. (a) Intensity transmission and phase-shift; (b) Phasor evolution in the complex plane. Only polarizers at $+45^{\circ}$ with respect to the $\mathrm{X}$-axis (neutral line).

In figure 3(a) we can see how we can implement the BIM just by selecting the appropriate gray levels that encode the ON and OFF state, in this case we select gray level 12 as OFF state, and gray level 239 as ON state. These levels are the ones that maximize the contrast. In figure 3(b) we can see how this BIM scheme can be performed with a PA-LCoS, there is a phase coupled with the different gray levels but, as we only use intensity, this is not an issue. In the experimental measurements we obtain that the low and high transmission points occur slightly displaced at gray levels 14 and 248, and the contrast we measure is about 1:50.

BIM provides us with an easy detection scheme: we just need to detect intensity. But, this modulation scheme produces a large DC term, that can saturate the dynamic range of the material ${ }^{[31][32]}$, reducing the number of multiplexed pages that can be stored in the material. There are some techniques that can be applied to reduce this DC term, such as pseudorandom phase mask ${ }^{[33]}$ or defocusing the recording plane ${ }^{[31][33]}$. A solution is to use another modulation scheme such as Hybrid Ternary Modulation (HTM). This scheme will be presented in the next subsection. 


\subsection{Hybrid Ternary Modulation}

The goal of Hybrid-Ternary Modulation (HTM) is to obtain a null DC-term maintaining the easy detection scheme from the BIM. HTM has been demonstrated and analysed with twisted-nematic $\operatorname{LCDs}^{[25]}$. We want to show in this section the problems and possible solutions when PA-LCoS devices are used to implement a HTM scheme.

For implementing HTM we need three gray level values, two of them with a high and equal transmission level, and with a $180^{\circ}$ relative phase-shift (these will be the ON levels), and a low intensity transmission level (OFF level). After performing a series of optimizations and simulations we have found that the PA-LCoS devices cannot accomplish these requirements. This is due to the fact that, regardless on the orientation of the polarizers or waveplates transmission axes, we always obtain a circular trajectory in the complex plane (as the ones showed in figure 3(b) and figure 4(b)), so the $180^{\circ}$ phase change is produced near the origin. In the case of twisted-nematic LCDs (TN-LCDs) it was possible, even though lowering the intensity transmission for the two ON levels ${ }^{[25]-[27][28]}$. In TN-LCDs amplitude modulation and phase-shift are coupled, which enabled to produce spiral and other arbitrary complex amplitude trajectories in the complex plane representation ${ }^{[28]}$.

In the case of PA-LCoS devices, if we want a good contrast ratio, we need that the low intensity level were closer to the origin as possible. Due to the circular trajectories involved, two equidistant points in the circle will not accomplish the requirement of a $180^{\circ}$ phase-shift difference, that it is produced in the origin. We found a compromise if the circular trajectory is shifted. Then at the cost of producing some leakage of light in the OFF value (lost of contrast ratio), we can produce two ON values with an appreciable intensity transmission and a relative phase-shift close to $180^{\circ}$. We can conclude that PA-LCoS devices cannot produce a pure HTM modulation scheme, but enable an approximate trade off, which we will call in the rest of the paper pseudo-HTM (pHTM). We want to study if the pHTM can be applied to a HDSS by simulating their performance in reducing the DC-term when compared with a pure binary intensity modulation.
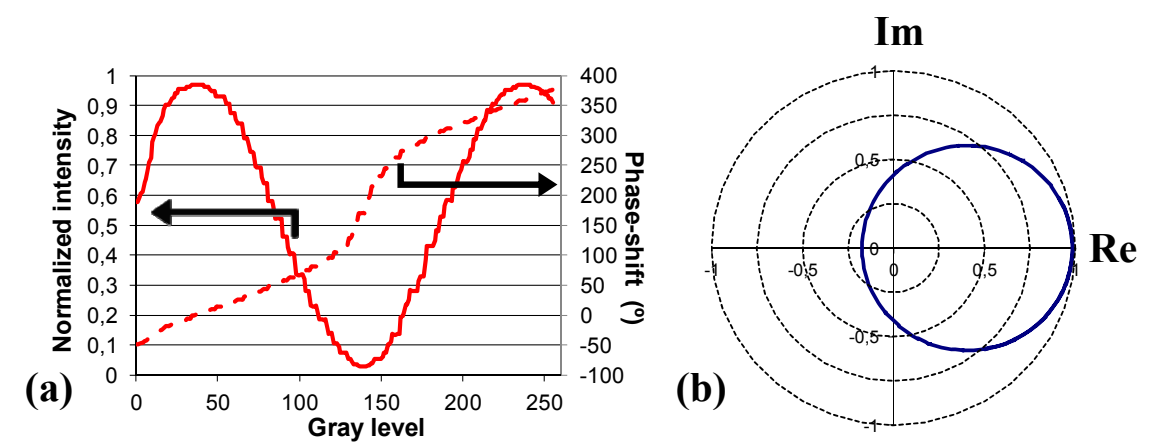

Fig. 4. Simulation for pHTM. (a) Intensity transmission and phase-shift; (b) Phasor evolution in the complex plane. Input and output polarizers at $+55^{\circ}$ and $-45^{\circ}$ with respect to the $\mathrm{X}$-axis.

In figure 4 we show the complex amplitude modulation for one of the possible pHTM configurations obtained in our simulations. These curves are obtained as in the case of BIM from the measured data in figure 2 (using the configuration for a full $2-\pi$ phase range) and the equation (4). In this case the input polarizer is rotated $55^{\circ}$ with respect the $\mathrm{X}$-axis and the output polarizer is at $-45^{\circ}$. In figure 4(b) we notice how the trajectory does not cross the origin, so the OFF level will imply some intensity transmission. The two ON gray levels considered are 105 and 168, with amplitude transmission values of 0.28 and phase-shift values of $75^{\circ}$ and $281^{\circ}$ respectively, i.e. a phase-shift difference of $206^{\circ}$. The OFF gray level is 140 with an amplitude transmission of 0.03 and phase-shift $170^{\circ}$. So the intensity contrast is 1:10.

In order to compare the reduction of the DC-term we will test this p-HTM configuration with a better contrast. To this goal we can configure PA-LCoS with the OFF gray level 140 (amplitude transmission of 0.03 and phase shift $170^{\circ}$ ) and the ON gray levels will be 69 and 207, which present an intensity transmission level of 0.76 and 0.77 , and a phase-shift of $33.3^{\circ}$ and $328.9^{\circ}$ respectively. In this case the intensity contrast is $1: 25$. This configuration will be labelled as p-HTM . 


\section{SIMULATION RESULTS}

In order to clarify the simulation results that are presented in this section, the data used for implementing the different modulation schemes are showed in Table 1.

Table 1. Modulation schemes used in the simulations. We can see the values used to implement the different modulation schemes presented in section 2. Note that BIM scheme only used one high level, HTM use the ideal values and the two pseudo-HTM use the data extracted from figure 4.

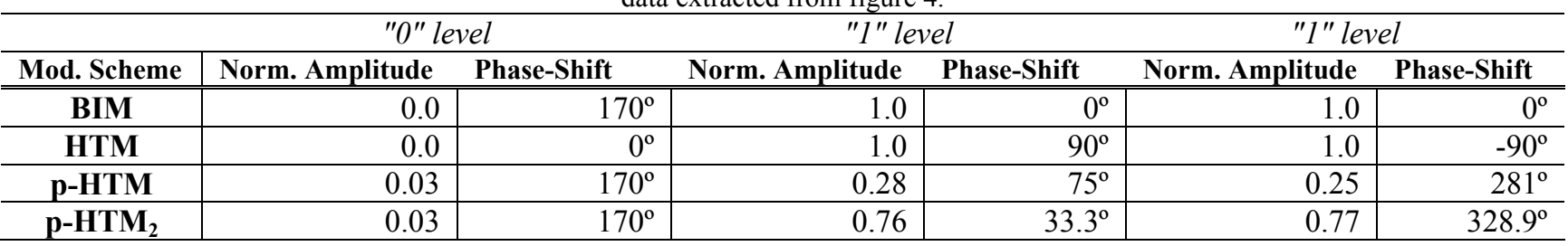

To numerically simulate the system we use the fast Fourier transform algorithm using the MATLAB software. In the simulations we consider data pages with $128 \times 128$ bits. To accurately simulate the optical system we have to consider both oversampling and zero padding of the data page bits ${ }^{[29]}$. According to all these factors, the size in pixel number of the Nyquist radius $R_{N}$ is 256 pixels.

In this work we are interested in evaluating the homogeneity of the intensity distribution in the recording plane. We consider the ratio between the DC-term (zero frequency) intensity value $I_{D C}$ with respect to the intensity $I_{\text {avrg }}$ averaged across the recording plane, given in decibels $(\mathrm{dB})$. This figure of merit is a variation of the ones defined in the works from $\mathrm{O}^{\prime}$ Callagan and Das ${ }^{[30][33]}$. We want to compare this relation for the four simulated modulation schemes: this means BIM, p-HTM, p-HTM 2 and HTM.

$$
R_{D C \text { term }}=10 \log _{10}\left(I_{D C} / I_{\text {avrg }}\right)
$$

Next we show some simulated results. We apply gaussian additive noise with a variance of 0.005 and mean zero, which is a good estimation of the thermal noise of a CCD camera that will be used in the detection phase. In the BIM we consider random bit data pages with an equal number of 0 s and $1 \mathrm{~s}$.

(a)

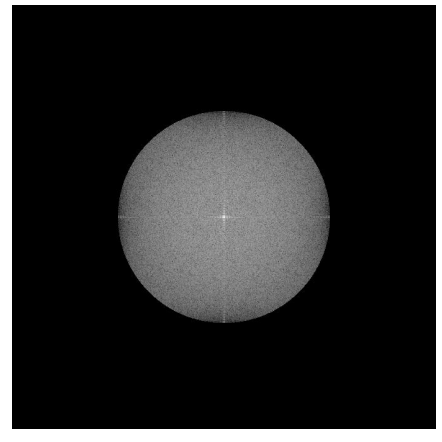

(b)

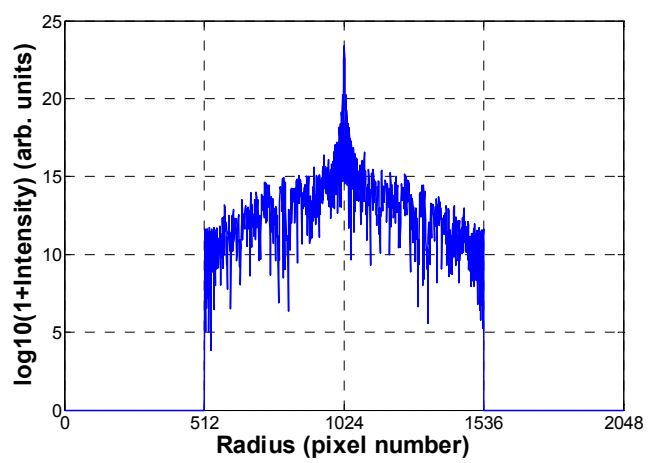

Fig. 5. Fourier plane where the limiting stop is double the length of the Nyquist radius. (a) Image plane; (b) Radial cut across a diameter crossing the center of the Fourier plane. The representation is logarithmic. We have considered a 128x128 bits BIM data page.

In Fig. 5 we show the resulting pattern in the Fourier plane for an ideal BIM data page, when considering a limiting stop with a length double the Nyquist radius, $R_{N}=256$ pixels, that imply an aperture diameter of 1024 pixels. Therefore, it 
transmits both the fundamental frequency and the first harmonic for the data page. We can see, especially in the radial cut showed in Fig. 5(b), the much larger intensity of the zero frequency (or DC term) in comparison with the rest of the frequency components in the Fourier plane. This is what happens when using BIM data pages and results in saturation of the dynamic range of the recording material in the location of the DC term, therefore deteriorating the data page information when retrieved and reducing the dynamic range of the recording material.

Next we are considering the pHTM scheme. We consider a random bit data page with an equal number of $0 \mathrm{~s}$ and $1 \mathrm{~s}$, where the $1 \mathrm{~s}$ are equally distributed between the two ON levels (with a phase-shift difference of $206^{\circ}$ ), (see Table 1).

(a)

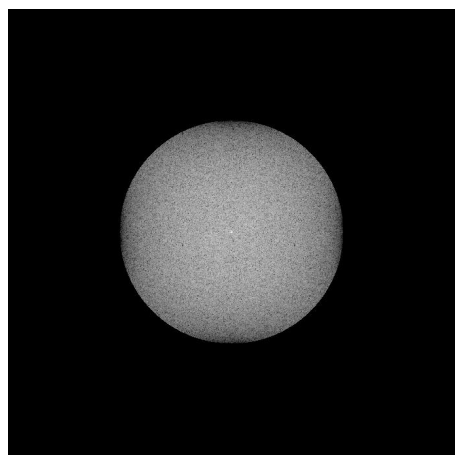

(b) $\underline{\square}$

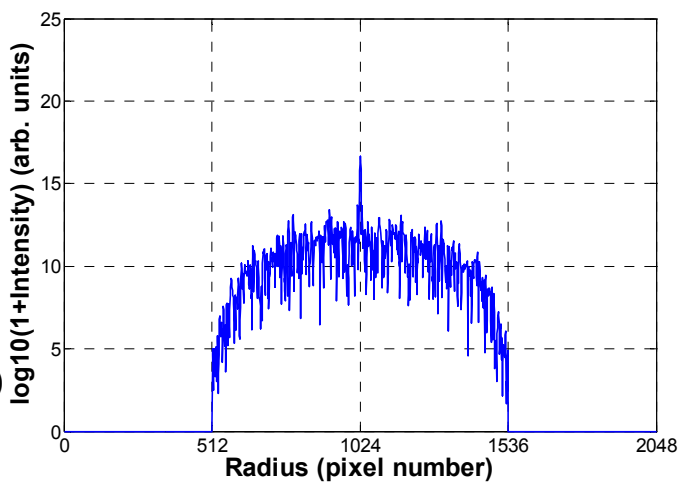

Fig. 6. Fourier plane where the limiting stop is double the length of the Nyquist radius. (a) Image plane; (b) Radial cut across a diameter crossing the center of the Fourier plane. The representation is logarithmic. We have considered a 128x128 bits p-HTM data page.

The results with the p-HTM scheme show us a clear reduction on the DC-Term, slightly above 15 (arb. units). In the image from the Fourier plane, it can be seen how the DC term is clearly reduced. For comparison we simulated the pure HTM scheme that it is show in figure 7.

(a)

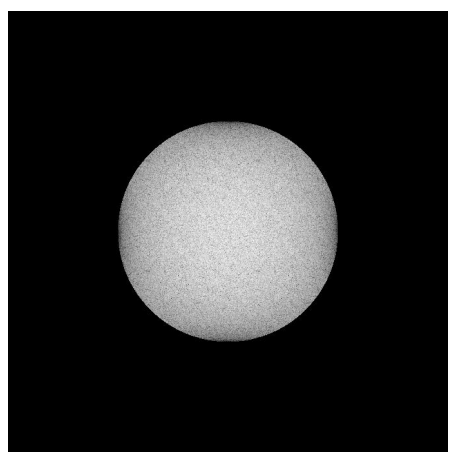

(b) $\underline{\underline{0}}$

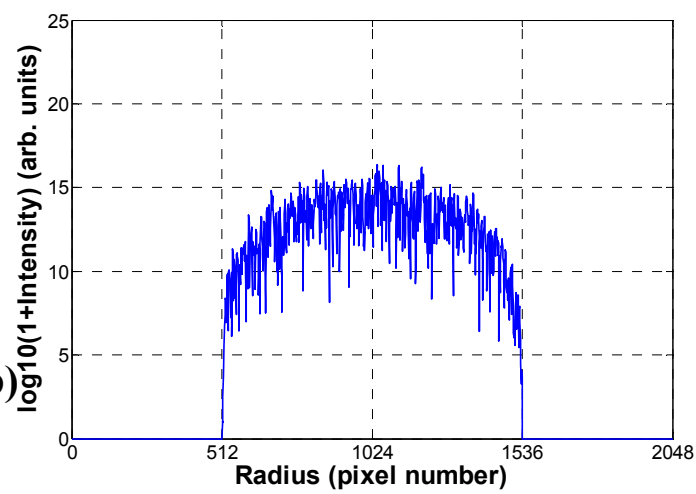

Fig. 7. Fourier plane where the limiting stop is double the length of the Nyquist radius. (a) Image plane; (b) Radial cut across a diameter crossing the center of the Fourier plane. The representation is logarithmic. We have considered a $128 \times 128$ bits HTM data page.

With the HTM scheme the DC-Term is missing, as expected, and the energy is better distributed along all the terms. As discussed in previous section this scheme cannot be achieved with PA-LCoS devices. In order to see how affects the phase-shift difference selected for $\mathrm{p}$-HTM scheme, now we simulate the $\mathrm{p}-\mathrm{HTM}_{2}$ scheme, where the contrast is augmented but the phase-shift between the two On-levels is far from the ideal $180^{\circ}$. The contrast is augmented from 1:10 to $1: 25$, and the phase-shift between the ON levels is $295.6^{\circ}$ (see Table 1). 
(a)
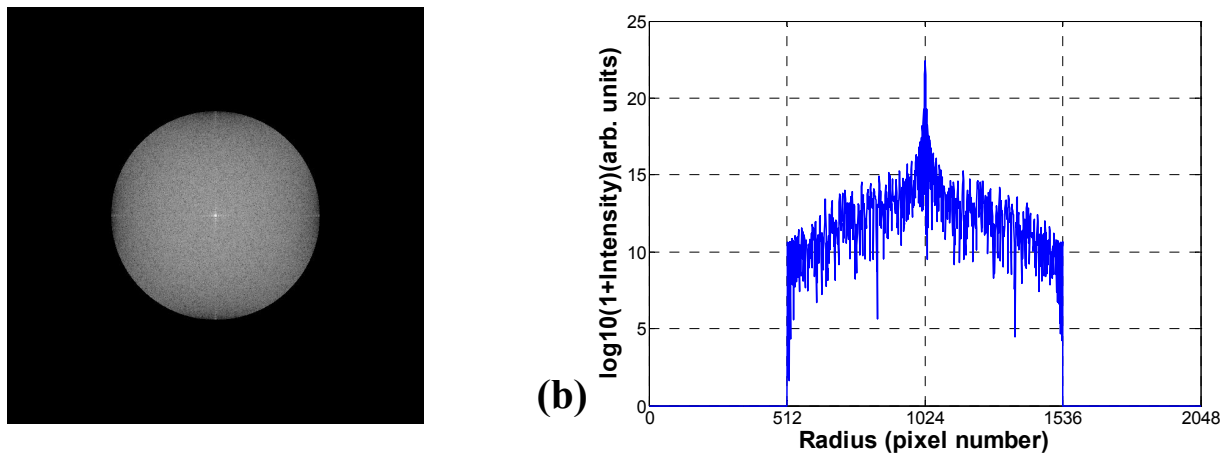

Fig. 8. Fourier plane where the limiting stop is double the length of the Nyquist radius. (a) Image plane; (b) Radial cut across a diameter crossing the center of the Fourier plane. The representation is logarithmic. We have considered a $128 \times 128$ bits p-HTM 2 data page.

In figure 8 we see how the $\mathrm{p}-\mathrm{HTM}_{2}$ scheme is closer to the BIM scheme. The DC-term is practically the same as BIM. This is due to the fact that the phase-shift between the ON levels is near $360^{\circ}$ (or $0^{\circ}$ ) i.e. far from $180^{\circ}$. So the better compromise, in order to reduce the DC-term, would be to use the configuration labeled as p-HTM.

Apart from the results presented, the figure of merit presented in equation (5) was calculated and we obtain the next results for $R_{D C t e r m}$.

Table 2. $R_{D \text { Cterm }}$ extracted from the different modulation schemes. $R_{D C \text { term }}$ is calculated following equation (5) and reflects the homogeneity of the fourier transform for different modulation schemes. A lower value implies a higher homogeneity.

\begin{tabular}{l|c} 
Modulation Scheme & $\boldsymbol{R}_{\text {DCterm }}(\mathbf{d B})$ \\
\hline \hline BIM & 51.01 \\
\hline HTM & 17.98 \\
\hline p-HTM & 33.54 \\
\hline p-HTM & 49.32 \\
\hline
\end{tabular}

We see in Table 2 how the $R_{D C \text { Cterm }}$ is reduced in HTM. For p-HTM $\mathrm{H}_{2}$ and BIM, it presents almost the same value. For pHTM the term is reduced in $16 \mathrm{~dB}$, so the DC-term is clearly diminished and this will facilitate us to prevent the saturation of the recording material for HDSS.

\section{CONCLUSIONS}

In this paper we have studied some modulation schemes implemented with a novel PA-LCoS device. We have found that we cannot achieve the HTM scheme that allows us to reduce the DC-term. The DC-term is an issue due to the effect in the saturation of the holographic material. The reduction of the DC-term while maintaining the easy detection of the BIM scheme, where only intensity needs to be detected, would be an important task when implementing a HDSS. For these reasons, we can conclude that the average Stokes polarimetry technique has provided us a proper mechanism to search for a modulation scheme to be used in PA-LCoS microdisplays. Even though HTM is not possible to be implemented in phase only devices, we have found a very good compromise. This solution is the so called p-HTM, that has been showed to reduce the DC-term, obtaining a performance between the ideal HTM and the BIM. This study enables us to use a PALCoS device as an entry data point in a holographic data storage system. 


\section{ACKNOWLEDGEMENTS}

This work was supported by the Ministerio de Trabajo y Competitividad of Spain under projects FIS2014-56100-C2-1-P and FIS2015-66570-P and by the Generalitat Valenciana of Spain (under projects PROMETEOII/2015/015 and ISIC/2012/013) and by the University of Alicante (GRE12-14).

\section{REFERENCES}

[1] J. Turunen and F. Wyrowski, eds., [Diffractive Optics for Industrial and Commercial Applications], Akademie Verlag (1997).

[2] H. J. Coufal, D. Psaltis, and B. T. Sincerbox, eds., [Holographic Data Storage], Springer-Verlag (2000).

[3] W. Osten, C. Kohler, and J. Liesener, "Evaluation and application of spatial light modulators for optical metrology," Opt. Pura Apl. 38, 71-81 (2005).

[4] M. A. F. Roelens, S. Frisken, J. A. Bolger, D. Abakoumov, G. Baxter, S. Poole, and B. J. Eggleton, "Dispersion trimming in a reconfigurable wavelength selective switch," J. Lightw. Technol. 26, 73-78 (2008).

[5] M. Salsi, C. Koebele, D. Sperti, P. Tran, H. Mardoyan, P. Brindel, S. Bigo, A. Boutin, F. Verluise, P. Sillard, M. Bigot-Astruc, L. Provost, and G. Charlet, "Mode-Division Multiplexing of $2100 \mathrm{~Gb} / \mathrm{s}$ Channels Using an LCOS-Based Spatial Modulator," J. Lightw. Technol. 30, 618-623 (2012).

[6] M. A. Solís-Prosser, A. Arias, J. J. M. Varga, L. Rebón, S. Ledesma, C. Iemmi, and L. Neves, "Preparing arbitrary pure states of spatial qudits with a single phase-only spatial light modulator," Opt. Lett. 38, 47624765 (2013).

[7] S. T. Wu and D. K. Yang, [Reflective Liquid Crystal Displays], John Wiley \& Sons Inc. (2005).

[8] N. Collings, T. Davey, J. Christmas, D. Chu, and B. Crossland, "The Applications and Technology of PhaseOnly Liquid Crystal on Silicon Devices," J. Display Technol. 7, 112-119 (2011).

[9] J. E. Wolfe and R. A. Chipman, "Polarimetric characterization of liquid-crystal-on-silicon panels," Appl. Opt. 45, 1688-1703 (2006).

[10] A. Hermerschmidt, S. Osten, S. Krüger, and Thomas Blümel, "Wave front generation using a phase-only modulating liquid-crystalbased micro-display with HDTV resolution," Proc. SPIE 6584, 65840E (2007).

[11] J. R. Moore, N. Collings, W. A. Crossland, A. B. Davey, M. Evans, A. M. Jeziorska, M. Komarčević, R. J. Parker, T. D. Wilkinson, and H. Xu, "The silicon backplane design for an LCOS polarization-insensitive phase hologram SLM," IEEE Photon. Techol. Lett. 20, 60-62 (2008).

[12] A. Lizana, I. Moreno, A. Márquez, C. Iemmi, E. Fernández, J. Campos, and M. J. Yzuel, " Time fluctuations of the phase modulation in a liquid crystal on silicon display: characterization and effects in diffractive optics," Opt. Express 16, 16711-16722 (2008).

[13] A. Lizana, I. Moreno, A. Márquez, E. Also, C. Iemmi, J. Campos, and M.J.Yzuel, "Influence of the temporal fluctuations phenomena on the ECB LCoS performance," Proc. SPIE 7442, 74420G-1 (2009).

[14] J. García-Márquez, V. López, A. González-Vega, and E. Noé, "Flicker minimization in an LCoS spatial light Modulator," Opt. Express 20, 8431-8441 (2012).

[15] G. Lazarev, A. Hermerschmidt, S. Krüger, and S. Osten, "LCOS Spatial Light Modulators: Trends and Applications," in Optical Imaging and Metrology: Advanced Technologies, W. Osten and N. Reingand, eds., (John Wiley \& Sons, 2012).

[16]F. J. Martínez, A. Márquez, S. Gallego, M. Ortuño, J. Francés, A. Beléndez, and I. Pascual, "Electrical dependencies of optical modulation capabilities in digitally addressed parallel aligned LCoS devices," Opt. Eng. 53, 067104 (2014).

[17] A. Márquez, F. J. Martínez, S. Gallego, M. Ortuño, J. Francés, A. Beléndez, and I. Pascual, "Classical polarimetric method revisited to analyse the modulation capabilities of parallel aligned liquid crystal on silicon displays," Proc. SPIE 8498, 84980L (2012).

[18]F. J. Martínez, A. Márquez, S. Gallego, J. Francés, and I. Pascual, "Extended linear polarimeter to measure retardance and flicker: application to LCoS devices in two working geometries," Opt. Eng. 53, 014105 (2014).

[19] C. Ramirez, B. Karakus, A. Lizana, and J. Campos, "Polarimetric method for liquid crystal displays characterization in presence of phase fluctuations," Opt. Express 21, 3182-3192 (2013). 
[20]F.J. Martínez, A. Márquez, S. Gallego, J. Francés, I. Pascual, and A. Beléndez, "Retardance and flicker modeling and characterization of electro-optic linear retarders by averaged Stokes polarimetry," Opt. Lett. 39, 1011-1014 (2014).

[21]F.J. Martínez, R. Fernández, A. Márquez, S. Gallego, M. L. Álvarez, I. Pascual and A. Beléndez. "Exploring binary and ternary modulations on a PA-LCoS device for holographic data storage in a PVA/AA photopolymer," Opt. Express 23, 20459-20479 (2015)

[22] F.J. Martínez, A. Márquez, S. Gallego, M. Ortuño, J. Francés, A. Beléndez, and I. Pascual, "Averaged Stokes polarimetry applied to evaluate retardance and flicker in PA-LCoS devices," Opt. Express 22, 15064-15074 (2014).

[23] G. Goldstein, [Polarized Light], Marcel Dekker (2003).

[24] T. G. Brown and Q. Zhan, "Focus Issue: Unconventional Polarization States of Light," Opt. Express 18, 10775-10776 (2010).

[25] J. S. Jang and D. H. Shin, "Optical representation of binary data based on both intensity and phase modulation with a twisted-nematic liquid-crystal display for holographic digital data storage," Opt. Lett. 26(22), 17971799 (2001)

[26] A. Márquez, S. Gallego, D. Méndez, M. L. Alvarez, E. Fernández, M. Ortuño, C. Neipp, A. Beléndez, and I. Pascual, "Accurate control of a liquid-crystal display to produce a homogenized Fourier transform for holographic memories," Opt. Lett, 32(17), 2511-2513 (2007)

[27] E. Fernández, A. Márquez, S. Gallego, R. Fuentes, C. García, and I. Pascual, "Hybrid ternary modulation applied to multiplexing holograms in photopolymers for data page storage," J. Lightwave Technol. 28(5), 776-783 (2010)

[28] A. Márquez, S. Gallego, D. Méndez, M. L. Álvarez, E. Fernández, M. Ortuño, A. Beléndez, and I. Pascual, "Characterization and optimization of liquid crystal displays for data storage application," Proc. SPIE 6587, 658715 (2007)

[29] A. Márquez, E. Fernández, F. J. Martínez, S. Gallego, M. Ortuño, A. Beléndez, and I. Pascual, "Analysis of the geometry of a holographic memory setup," Proc. SPIE 8429, 84291Y (2012)

[30] M. J. O'Callaghan, "Sorting through the lore of phase mask options - performance measures and practical commercial desing," Proc. SPIE 5362, 159 (2004)

[31]B. Das, J. Joseph, and K. Singh, "Material saturation in photopolymer holographic data recording and its effects on bit-error-rate and content-addressable search," Opt. Pura Apl. 42, 125-132 (2009)

[32] E. Fernández, A. Márquez, M. Ortuño, R. Fuentes, C. García, and I. Pascual, "Optimization of twistednematic liquid crystal displays for holographic data storage," Opt. Pura Apl. 42, 125-132 (2009)

[33]B. Das, J. Joseph, and K. Singh, "Performance analysis of content-addressable search and bit-error rate characteristics of a defocused volume holographic data storage system," Appl. Opt. 46(22), 5461-5470 (2007) 